\title{
Development and validation of a nomogram for predicting the status of estrogen receptor-low-positive breast cancer
}

\author{
Zhanli Jia ${ }^{1 \#}$, Shuyao Niu ${ }^{1 \#}$, Xinle Wang ${ }^{2}, \mathrm{Si} \mathrm{Wu}^{1}$, Jinze $\mathrm{Li}^{1}$, Mengxue Han ${ }^{1}$, Yueping Liu ${ }^{1}$ \\ ${ }^{1}$ Department of Pathology, The Fourth Hospital of Hebei Medical University, Shijiazhuang, China; ${ }^{2}$ Department of Breast Center, The Fourth \\ Hospital of Hebei Medical University, Shijiazhuang, China \\ Contributions: (I) Concept and design: Z Jia, Y Liu; (II) Administrative support: Y Liu; (III) Provide learning materials or patients: S Niu, S Wu; (IV) \\ Collect and assemble data: Z Jia, S Niu, J Li; (V) Data analysis and interpretation: Z Jia, S Niu, M Han; (VI) Manuscript writing: All authors; (VII) \\ Final approval of the manuscript: All authors. \\ "These authors contributed equally to this work. \\ Correspondence to: Dr. Yueping Liu. Department of Pathology, the Fourth Hospital of Hebei Medical University, No. 12 Jiankang Road, Shijiazhuang \\ 050011, China. Email: annama@163.com.
}

Background: To identify whether patients with estrogen receptor (ER)-low-positive breast cancer can benefit from endocrine therapy, it is of great significance to guide their treatment. We have developed and validated a nomogram to predict the status of ER-low-positive breast cancer based on clinicopathological characteristics.

Methods: A retrospective study was conducted on 450 patients with invasive breast cancer. Among them, 139 (30.89\%) patients were ER-negative (ER < 1\%) and 311 patients (69.11\%) were ER-positive (ER > 10\%), and the two groups of patients were randomly divided into training cohort and validation cohort at a ratio of 7:3. Multivariate logistic regression analysis was performed, after which a nomogram for predicting the status of ER was constructed and validated. Discrimination of the prediction model was assessed using an area under the receiver operating characteristic curve (AUC), and calibration was assessed using calibration plots. The Youden index was calculated to determine the optimal cut-off value. Finally, the nomogram is used to predict the status of 260 ER-low-positive (1-10\%) breast cancer.

Results: The final multivariate regression model included nuclear pleomorphism, mitoses, TILs, and necrosis. The nomogram showed good calibration and discrimination in both sets (an AUC of 0.804 and 0.828 for the training and validation cohorts, respectively). According to the Youden index, the optimal cutoff value was 0.59 . Status prediction was performed for ER-low-positive breast cancer, of whom $63.08 \%$ was predicted as negative and $36.92 \%$ was predicted as positive. Those who with negative predicted result had lower levels of ESR1 mRNA expression, did not benefit from endocrine therapy, and had a poorer prognosis. Conclusions: We developed and validated a nomogram to predict the status of ER-low-positive breast cancer based on clinicopathological characteristics. The nomogram will facilitate individualized precision treatment of ER-low-positive breast cancer.

Keywords: Breast cancer; estrogen receptor low positive (ER low positive); nomogram

Received: 15 June 2021; Accepted: 12 July 2021; Published: 31 July 2021.

doi: $10.21037 /$ tbcr-21-12

View this article at: https://dx.doi.org/10.21037/tbcr-21-12 


\section{Introduction}

According to the 2018 Global Cancer Statistics, breast cancer is the most common cancer and the leading cause of cancer death among women (1). The expression of estrogen receptor (ER) is of crucial significance to guiding treatment and predicting the prognosis of breast cancer patients (2-4). Although $1 \%$ is used as the cutoff value for positive ER expression and endocrine therapy is recommended (5), in the 2020 update of the American Society of Clinical Oncology/College of American Pathologists (ASCO/CAP) guideline for the immunohistochemical testing of ERs in breast cancer, the expert panel acknowledges that there are limited data on endocrine therapy benefit for cancers with $1 \%$ to $10 \%$ of cells staining ER positive, and that a new reporting category should be used: ER low positive (6). A number of retrospective studies (7-21) found that ER-low-positive breast cancer is a heterogeneous group of diseases in which most patients share similar clinicopathological features, molecular characteristics, and clinical prognosis as those with ER-negative tumors, and do not benefit from endocrine therapy. Despite the relatively low toxic side effects of endocrine therapy, the correct identification of patients who would not benefit from endocrine therapy among ER-low-positive breast cancer is great importance to guiding the treatment.

Although ER-low-positive tumors account for only $2-3 \%$ of all ER-positive tumors, a special clinical challenge exists for this group of patients, which is the issue of how they should be treated (6). In ER-low-positive breast cancer, the effect and prognosis of endocrine therapy in patients cannot be accurately assessed based on the expression level of ER alone. Therefore, we developed a nomogram based on the patients' clinicopathological characteristics, which can serve as a method to predict the status of ER-low-positive breast cancers, in order to guide the individualized precision treatment of ER-low-positive patients. We present the following article in accordance with the TRIPOD reporting checklist (available at https:// dx.doi.org/10.21037/tbcr-21-12).

\section{Methods}

\section{Patients}

The database of the Department of Pathology of the Fourth Hospital of Hebei Medical University was reviewed, from which 450 patients with primary invasive breast cancer were screened (Figure 1). The patients underwent surgery at the Fourth Hospital of Hebei Medical University from January 2012 to December 2012, of which 139 was ER-negative $(<1 \%)$ and 311 was ER-positive (>10\%). The two groups of patients were randomly divided into training cohort and validation cohort at a ratio of 7:3.

In addition, 260 patients with ER-low-positive (1-10\%) primary invasive breast cancer, who underwent surgery at the Fourth Hospital of Hebei Medical University from January 2012 to December 2018, were also screened.

$1-100 \%$ tumor cell nuclear staining is defined as ER positive, and for ER 1-10\% tumor cell nuclear staining, it is defined as ER low positive. If $<1 \%$ of tumor cells have nuclear staining, the ER is considered negative.

\section{Statistics}

Clinicopathological characteristics include the following factors: age, menopausal status, primary site, histological type, tumor size, lymph node status, clinical stage, LVI, tubule formation, nuclear pleomorphism, mitoses, histological grade, TILs, necrosis, and ER. Univariate logistic regression was used to test the associations between ER and clinicopathological characteristics. Multivariable logistic regression was performed to identify independent covariates. In the final nomogram, only factors with $\mathrm{P}<0.05$ were retained.

The performance of the nomogram was quantified with respect to discrimination and calibration. The receiver operating characteristic (ROC) curve was drawn, and the predicted accuracy was assessed by calculating the area under the ROC curve (AUC). When the AUC is above 0.75 , the model is considered to have good predictive performance. A $95 \%$ CI was calculated for each AUC. Calibration was used to illustrate the relation between the predicted and observed probabilities. Youden index was used to determine the optimal cut-off value of the nomogram.

Statistical analyses were performed using $\mathrm{R}$ version 3.6.3, RStudio version 1.3.1093, GraphPad Prism version 8.0.1.

\section{Measures and assessment of ER status}

The nomogram was extrapolated to ER-low-positive tumors to obtain the predicted results. Total RNA extraction was performed on each ER-low-positive tumor sample, and the expression level of ESR1 mRNA was detected using onestep RT-qPCR. To exclude the possibility of heterogeneity in tumor tissues, ER immunohistochemistry and ESR1 
gene expression levels were measured on the same paraffinembedded tissue block for each sample. The differences in ESR1 mRNA expression levels and prognosis were compared between the two groups of patients with negative and positive predicted results.

This study was conducted in accordance with the Declaration of Helsinki (as revised in 2013) and the Harmonized Tripartite Guideline for Good Clinical Practice from the International Conference on Harmonization. This study was approved by Institutional Review Board of The Fourth Hospital of Hebei Medical University. Informed consent was obtained from all individual participants included in the study.

\section{Results}

\section{Patient characteristics}

In this research, data were retrospectively collected from 450 patients with invasive breast cancer, including 139 (30.89\%) ER-negative (<1\%) cases and 311 (69.11\%) ERpositive $(>10 \%)$ cases. The two groups were randomly divided into the training and validation cohorts at a ratio of 7:3. The two cohorts showed no statistically significant differences for any of the clinicopathological characteristics ( $\mathrm{P}>0.05)$, which implied that the data of the two groups were comparable. The clinicopathological characteristics of the research population are shown in Table 1 .

\section{Predictors associated with ER expression}

Since the purpose of our study was to explore the effects of the patients' clinicopathological characteristics of tumor tissues on ER expression, we did not include immunohistochemical indicators in our analysis. Furthermore, given that correlations of tubule formation, nuclear pleomorphism, and mitoses with histological grade may reduce the accuracy of the nomogram, we eliminated histological grade from the logistic regression analysis.

In the univariate logistic regression analysis of the training cohort, factors associated with ER expression included primary site $(\mathrm{P}=0.01)$, histological type $(\mathrm{P}=0.01)$, TILs $(\mathrm{P}<0.001)$, nuclear pleomorphism $(\mathrm{P}<0.001)$, mitoses $(\mathrm{P}<0.001)$, and necrosis $(\mathrm{P}<0.001)$. Multivariate logistic regression analysis was then performed, and independent factors associated with ER expression included nuclear pleomorphism $(\mathrm{P}=0.0339)$, mitoses $(\mathrm{P}=0.0022)$, TILs $(\mathrm{P}<0.001)$, and necrosis $(\mathrm{P}=0.0007)$ (Table 2).

\section{Development of the nomogram}

Based on the independent factors identified by multivariate logistic regression analysis, the patients' clinicopathological characteristics of tumor tissues were used to develop a nomogram that included nuclear pleomorphism, mitoses, TILs, and necrosis in order to predict ER expression (Figure 2).

\section{Validation of the nomogram}

Firstly, we evaluate the consistency of the nomogram. In the training cohort (Figure $3 A$ ) and the validation cohort (Figure $3 B$ ), the calibration plots of the nomogram show a good consistency between the predicted probability and the actual probability. The AUCs of the training cohort (Figure 4A) and the validation cohort (Figure $4 B$ ) were 0.804 (95\% CI: $0.750-0.858$ ) and 0.828 (95\% CI: 0.752-0.903), respectively. The AUC values of the training cohort and the validation cohort are similar, which indicates that the nomogram does not over fit the training data, so it has good prediction ability.

\section{Determining the cut-off value for predicting ER expression}

A higher cut-off value would result in increased specificity and decreased sensitivity, whereas a lower cut-off value would result in increased sensitivity and decreased specificity. By calculating the Youden index, we derived an optimal cut-off value of 0.59 for predicting ER expression that ensured both sensitivity and specificity (Figure $4 A$ ). The prediction value $<0.59$ indicates a negative $\mathrm{ER}$ result, and the prediction value $>0.59$ indicates a positive ER result.

\section{Dynamic nomogram}

In order to improve the applicability of the nomogram to clinical practice, a dynamic nomogram was constructed based on the model established by R language (Figure 5). Using the nomogram, the predicted values for ER expression can be calculated by evaluating nuclear pleomorphism, mitoses, TILs, and necrosis.

\section{Status prediction for patients with ER-low-positive tumors}

Status prediction was performed for 260 ER-low-positive patients between 2012-2018 based on the dynamic nomogram. Our findings indicated that 164 patients $(63.08 \%)$ 
Table 1 Clinicopathological characteristics of patients in the training cohort and validation cohort

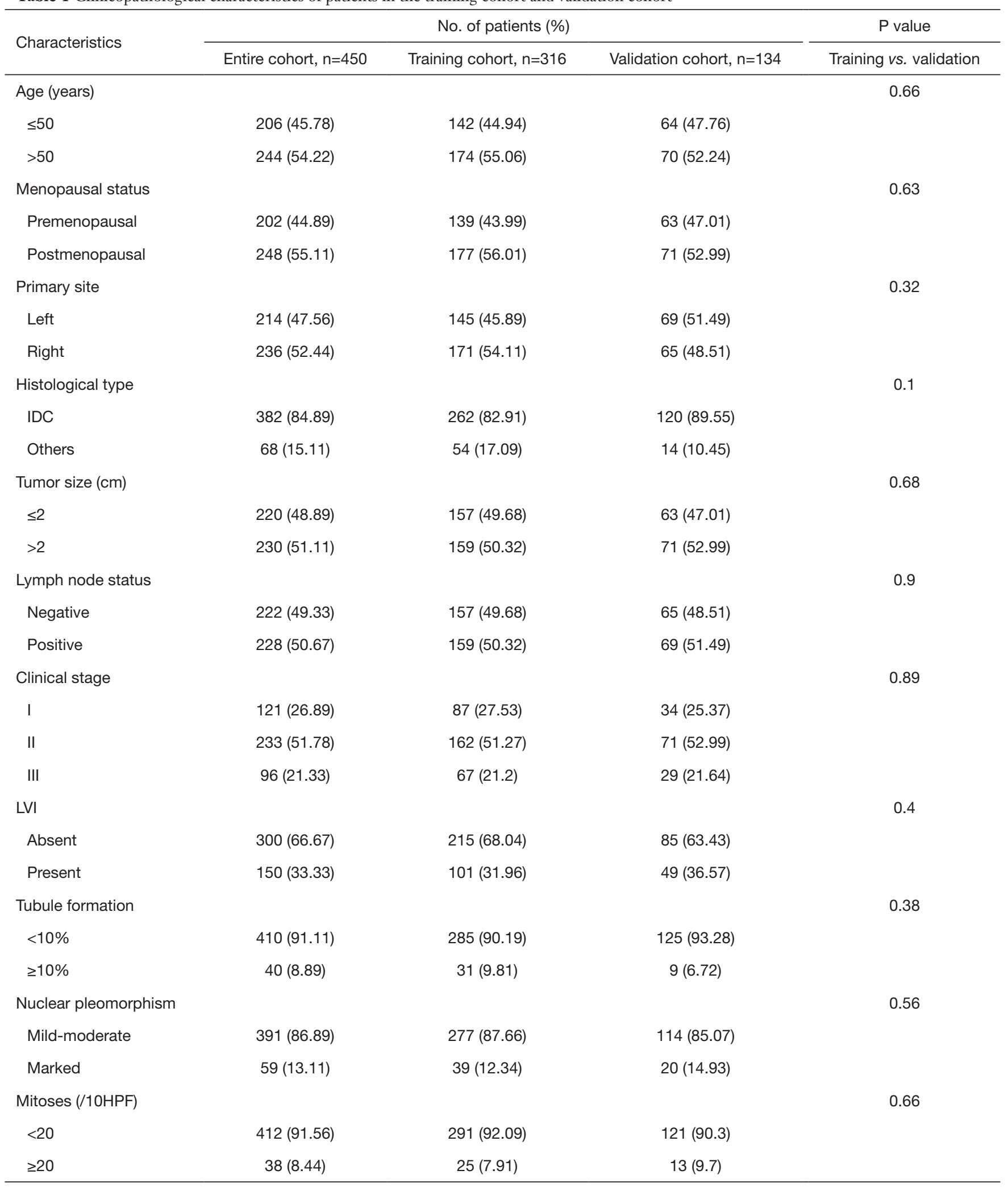

Table 1 (continued) 
Table 1 (continued)

\begin{tabular}{|c|c|c|c|c|}
\hline Characteristics & \multicolumn{3}{|c|}{ No. of patients (\%) } & $\begin{array}{c}\mathrm{P} \text { value } \\
\text { Training vs. validation }\end{array}$ \\
\hline Histological grade & & & & 0.07 \\
\hline I & 37 (8.22) & $31(9.81)$ & $6(4.48)$ & \\
\hline ॥ & $338(75.11)$ & 238 (75.32) & $100(74.63)$ & \\
\hline TILs & & & & 0.97 \\
\hline$\leq 40 \%$ & $374(83.11)$ & 262 (82.91) & $112(83.58)$ & \\
\hline$>40 \%$ & $76(16.89)$ & 54 (17.09) & $22(16.42)$ & \\
\hline Necrosis & & & & 0.79 \\
\hline ER & & & & 1 \\
\hline Negative & 139 (30.89) & $98(31.01)$ & $41(30.6)$ & \\
\hline Positive & $311(69.11)$ & 218 (68.99) & $93(69.4)$ & \\
\hline
\end{tabular}

ER, estrogen receptor.

Table 2 Univariate and multivariate logistic regression analysis of the training cohort

\begin{tabular}{|c|c|c|c|c|}
\hline Characteristics & \multicolumn{2}{|c|}{ Univariate analysis } & \multicolumn{2}{|c|}{ Multivariate analysis } \\
\hline Age & -0.30 & 0.22 & & \\
\hline Clinical stage & -0.19 & 0.65 & & \\
\hline Histological type & -0.80 & $0.01^{*}$ & & \\
\hline Lymph node status & 0.26 & 0.29 & & \\
\hline Menopausal status & -0.43 & 0.08 & & \\
\hline Mitoses & -4.25 & $<0.001^{*}$ & -3.30 & $0.0022^{*}$ \\
\hline Necrosis & -1.06 & $<0.001^{*}$ & -1.02 & $0.0007^{*}$ \\
\hline TILs & -2.69 & $<0.001^{*}$ & -2.09 & $<0.001^{*}$ \\
\hline Tubule formation & -16.92 & 0.98 & & \\
\hline Tumor size & -0.46 & 0.06 & & \\
\hline
\end{tabular}

${ }^{*} \mathrm{P}<0.05$. 


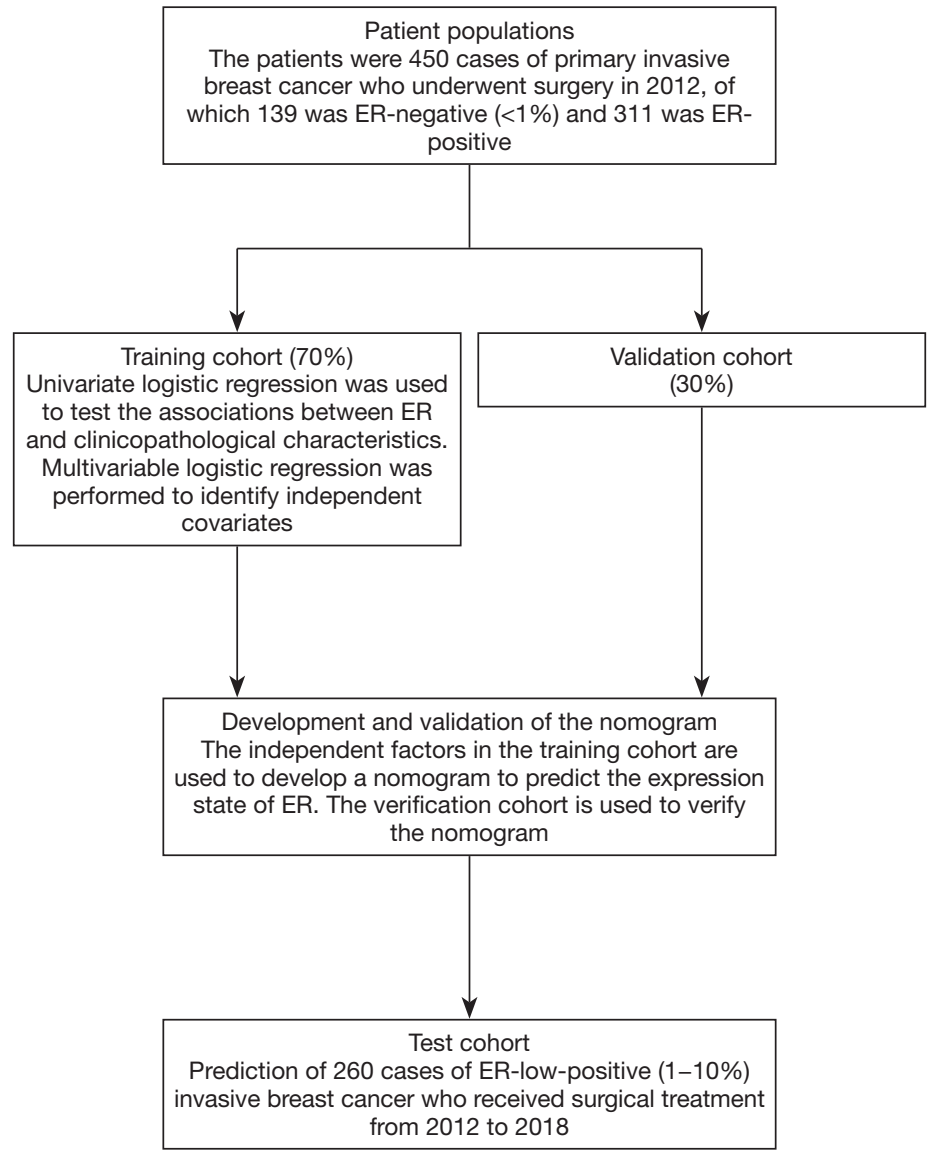

Figure 1 Study design. ER, estrogen receptor.

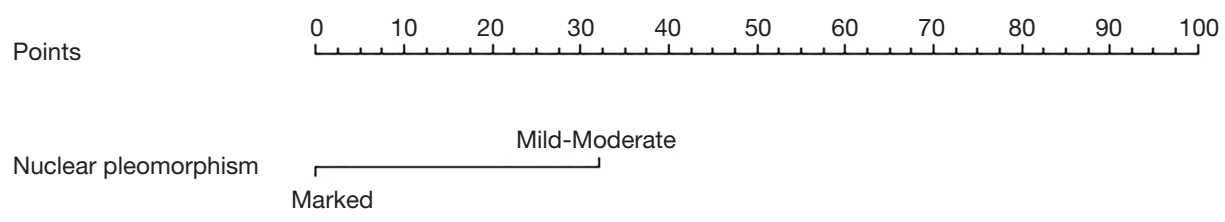

Mitoses

Marked

$$
\geq 20
$$

TILs

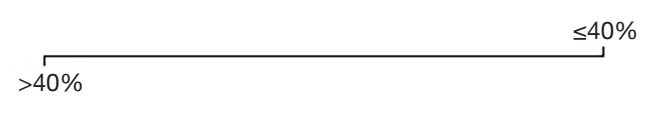

Necrosis

Absent

$$
\text { Present }
$$

Total Points

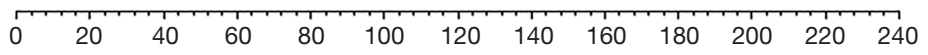

Predicted Value

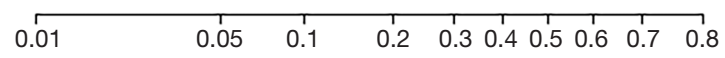

Figure 2 Nomogram for predicting ER expression. ER, estrogen receptor. 

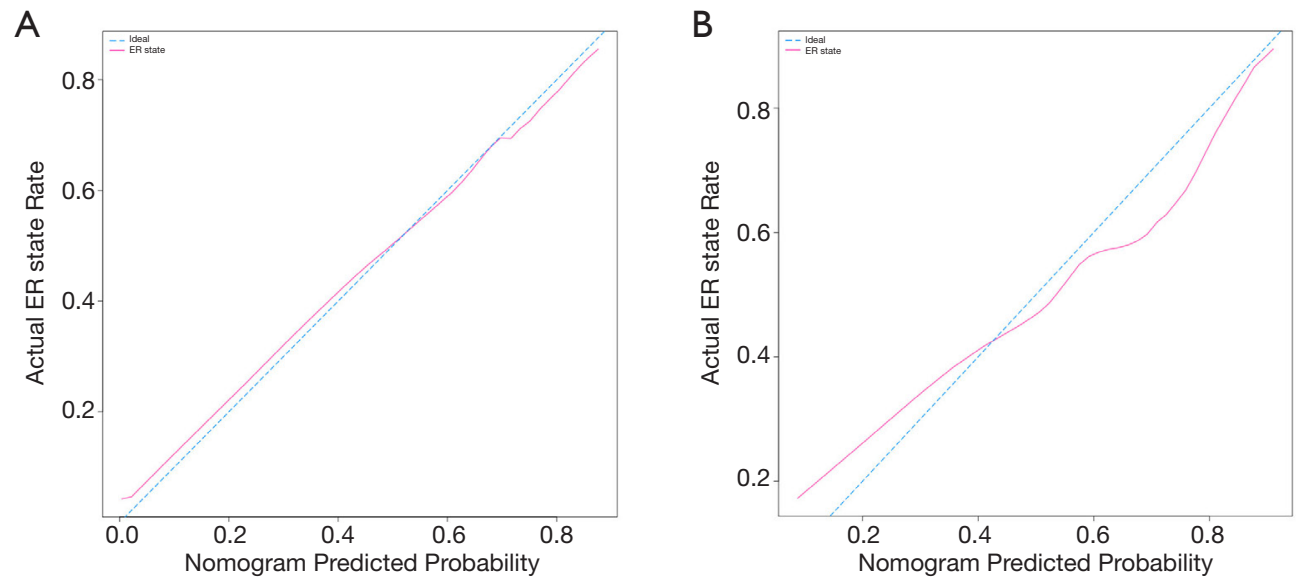

Figure 3 Calibration plots for the training cohort (A) and validation cohort (B). ER, estrogen receptor.

A

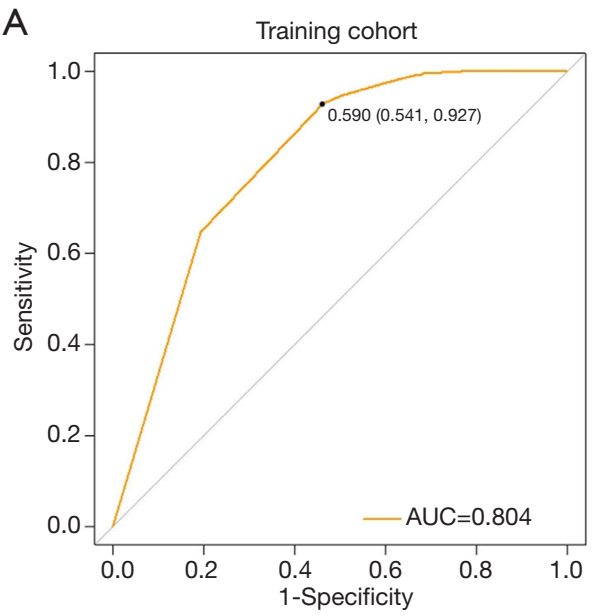

B

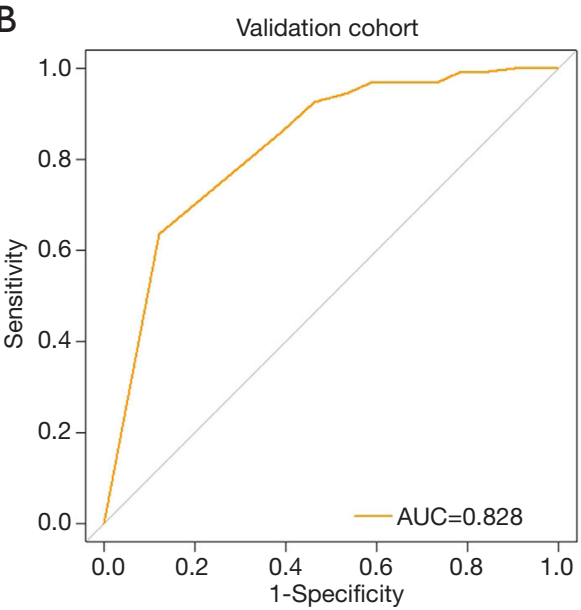

Figure 4 ROC curves for the training cohort (A) and the validation cohort (B). ROC, receiver operating characteristic; AUC, area under the ROC curve.

were predicted as negative (predicted value $<0.59$ ) and 96 $(36.92 \%)$ were predicted as positive (predicted value $>0.59$ ).

The expression level of ESR1 mRNA in each ER-lowpositive tumor sample was tested, and the Ct value of the ESR1 gene in each sample was normalized relative to the $\mathrm{Ct}$ value of the reference gene to obtain a $\Delta \mathrm{Ct}$ value. Patients with negative predicted results showed significantly lower expression levels of ESR1 mRNA $(\mathrm{P}<0.05)$ (Figure 6).

Survival analysis was performed on patients with ERlow-positive breast cancer in both groups based on their prediction results with a follow-up time of 24-106 months. In the overall population, patients with negative predicted results had significantly worse disease-free survival (DFS) $\left(\chi^{2}=13.040, \mathrm{P}<0.001\right)$ and overall survival $(\mathrm{OS})$ $\left(\chi^{2}=13.938, \mathrm{P}<0.001\right)$. To exclude the effect of anti-HER2 targeted therapy on prognosis, HER2-negative patients were selected for analysis, which showed that patients with negative predicted results had significantly worse DFS $\left(\chi^{2}=5.363, \mathrm{P}=0.021\right)$ and $\mathrm{OS}\left(\chi^{2}=5.761, \mathrm{P}=0.016\right)$. In addition, to examine whether patients in both groups would benefit from endocrine therapy, patients who received endocrine therapy were selected for analysis, and the results showed that patients with negative predicted results had significantly worse DFS $\left(\chi^{2}=4.613, \mathrm{P}=0.032\right)$ and OS $\left(\chi^{2}=5.069, \mathrm{P}=0.024\right)$ (Figure 7). These findings suggest that among patients with ER-low-positive breast cancer, those with a negative predicted result had a significantly worse prognosis and did not benefit from endocrine therapy. 


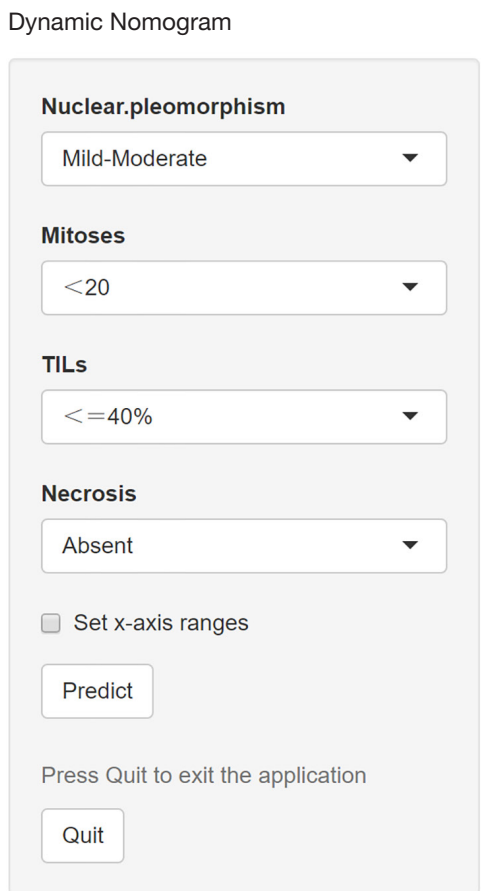

Figure 5 Dynamic nomogram.

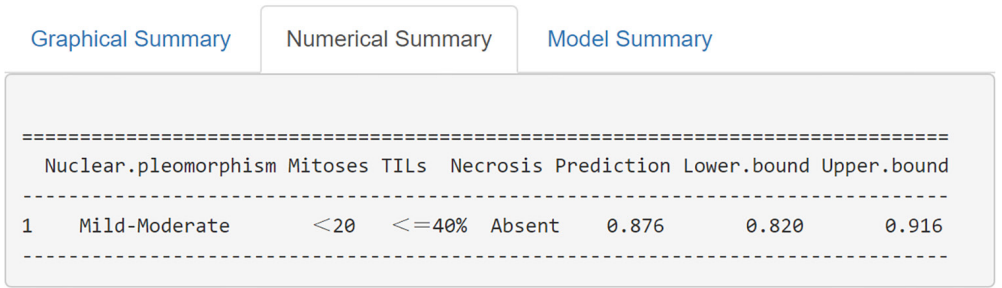

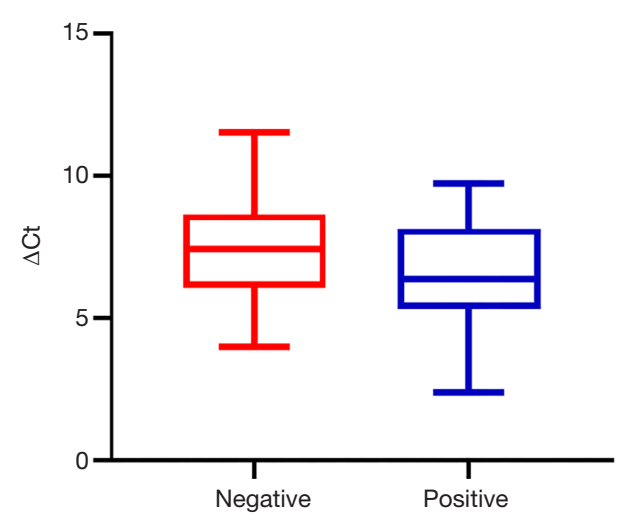

Figure 6 Expression of ESR1 mRNA in ER-low-positive tumors. $\mathrm{ER}$, estrogen receptor.

\section{Discussion}

ER-low-positive breast cancers are relatively rare, accounting for only $2-3 \%$ of ER-positive tumors (6). Thus, it is unlikely that prospective clinical trials will be conducted to determine the optimal treatment strategy for this status. Although endocrine therapy has relatively low toxic side effects, the proper identification of patients with
ER-low-positive breast cancer who will not benefit from endocrine therapy can reduce unnecessary treatments and toxic side effects, which is great importance to formulating patient treatment plans, improving their quality of life, and decreasing their financial burden.

Previous studies have shown that ER expression is associated with features of good prognosis, whereas ERnegative tumors are poorly differentiated and have a high nuclear grade, that is, ER-negative tumors have larger nuclei, show greater pleomorphism, and exhibit mitosis more frequently $(7,22)$. Moreover, in-depth investigations of TILs found that the distribution of TILs varied among breast cancer subtypes, with the highest levels found especially in triple-negative and HER2-overexpressing subtypes, and significantly lower levels found in hormone receptor positive breast cancers $(23,24)$. In summary, these findings suggest that in most cases, the clinicopathological features of tumor tissues are closely related to the ER expression.

To better guide the clinical development of individualized treatment plans for patients with ER-low-positive breast cancer, we developed a nomogram based on the clinicopathological characteristics, which can serve as a 

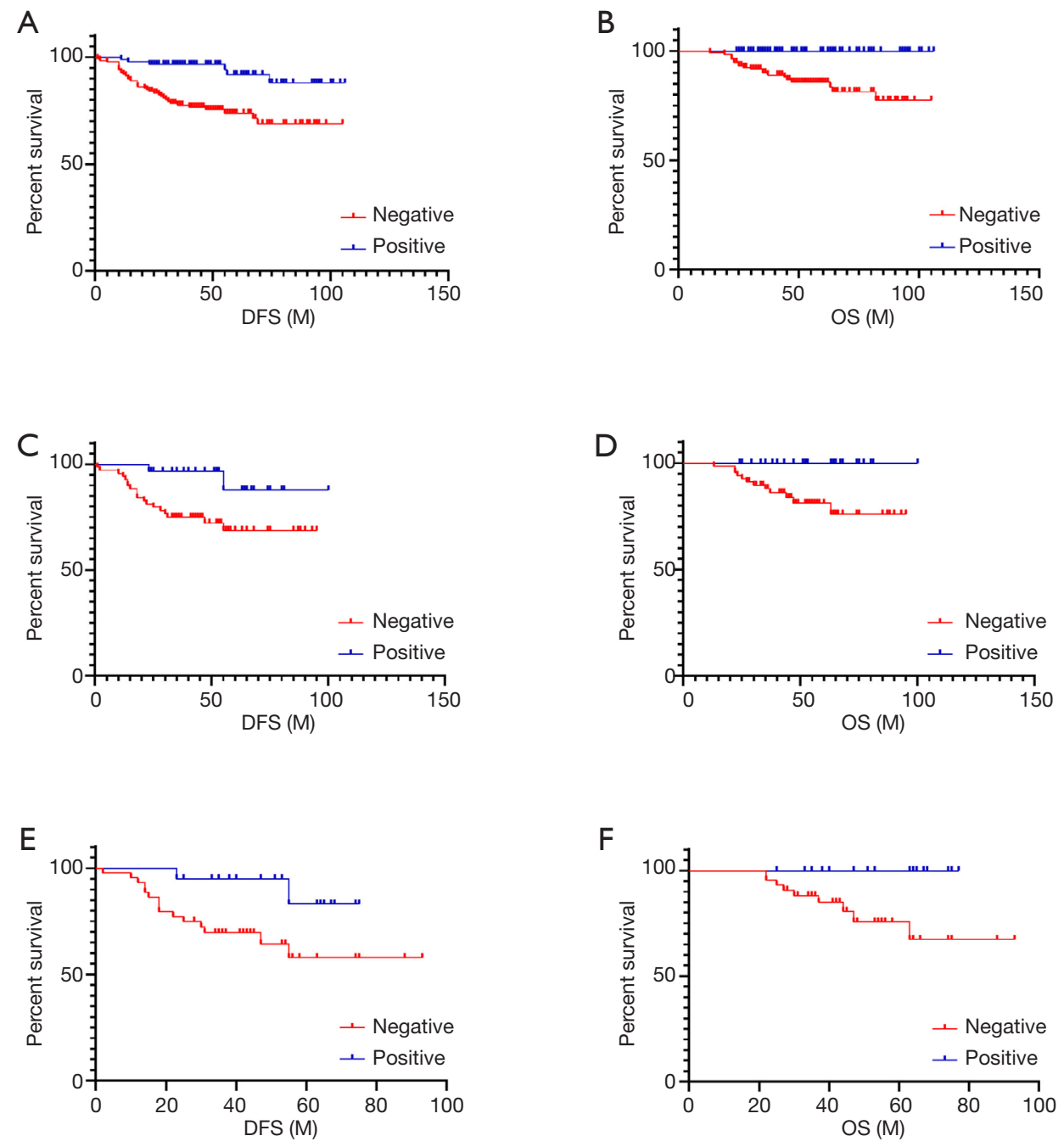

Figure 7 Survival analysis. (A,B) Survival analysis of patients with ER-low-positive breast cancer; (C,D) survival analysis of patients with ER-low-positive/HER2-negative breast cancer; (E,F) survival analysis of patients with ER-low-positive/HER2-negative breast cancer after endocrine therapy. DFS, disease-free survival; OS, overall survival; ER, estrogen receptor.

method to predicting the status of ER-low-positive breast cancer. Our study is consistent with the results of previous retrospective studies, which showed that the majority of ERlow-positive tumors exhibited similar clinicopathological features to ER-negative tumors, and that this group of patients did not benefit from endocrine therapy and had a poorer prognosis.

In addition, for resource-poor areas, prediction models may serve as a cheaper and more convenient alternative to immunohistochemical or genetic testing. However, there are still some limitations to this study. First, our study data came from the same institution, and did not take into account the differences between different ethnic groups, geographical areas, and other aspects. Second, our model only incorporated the morphological characteristics of tumor cells within the region of invasive carcinoma, and did not consider the effects of factors such as regions beyond the invasive carcinoma and tumor stromal cells on ER expression. These characteristics may help to optimize the model and enhance the accuracy in the predicted performance of the model.

\section{Conclusions}

First, the nomogram constructed by clinicopathological characteristics showed good discrimination of ER 
expression. Patients with significant nuclear pleomorphism, mitoses $>20 / 10 \mathrm{HPF}$, TILs $>40 \%$, and visible necrosis had a greater tendency to be negative for ER expression. Second, we applied the dynamic nomogram for the status prediction of ER-low-positive breast cancer, and the results showed that patients with predicted values $<0.59$ had lower ESR1 mRNA expression levels, did not benefit from endocrine therapy, and had a poorer prognosis. The nomogram can facilitate the accurate identification of these patients for whom ineffective endocrine therapy should be avoided in favor of more aggressive treatment strategies.

\section{Acknowledgments}

Funding: None.

\section{Footnote}

Reporting Checklist: The authors have completed the TRIPOD reporting checklist. Available at https://dx.doi. org/10.21037/tbcr-21-12

Data Sharing Statement: Available at https://dx.doi. org/10.21037/tbcr-21-12

Conflicts of Interest: All authors have completed the ICMJE uniform disclosure form (available at https://dx.doi. org/10.21037/tbcr-21-12). YL serves as the unpaid editorial board member of Translational Breast Cancer Research. The other authors have no conflicts of interest to declare.

Ethical Statement: The authors are accountable for all aspects of the work in ensuring that questions related to the accuracy or integrity of any part of the work are appropriately investigated and resolved. This study was conducted in accordance with the Declaration of Helsinki (as revised in 2013) and the Harmonized Tripartite Guideline for Good Clinical Practice from the International Conference on Harmonization. This study was approved by Institutional Review Board of The Fourth Hospital of Hebei Medical University. Informed consent was obtained from all individual participants included in the study.

Open Access Statement: This is an Open Access article distributed in accordance with the Creative Commons Attribution-NonCommercial-NoDerivs 4.0 International License (CC BY-NC-ND 4.0), which permits the noncommercial replication and distribution of the article with the strict proviso that no changes or edits are made and the original work is properly cited (including links to both the formal publication through the relevant DOI and the license). See: https://creativecommons.org/licenses/by-nc-nd/4.0/.

\section{References}

1. Bray F, Ferlay J, Soerjomataram I, et al. Global cancer statistics 2018: GLOBOCAN estimates of incidence and mortality worldwide for 36 cancers in 185 countries. CA Cancer J Clin 2018;68:394-424.

2. Burstein HJ, Temin S, Anderson H, et al. Adjuvant endocrine therapy for women with hormone receptorpositive breast cancer: american society of clinical oncology clinical practice guideline focused update. J Clin Oncol 2014;32:2255-69.

3. Albert JM, Gonzalez-Angulo AM, Guray M, et al. Patients with only 1 positive hormone receptor have increased locoregional recurrence compared with patients with estrogen receptor-positive progesterone receptorpositive disease in very early stage breast cancer. Cancer 2011;117:1595-601.

4. Bartlett JM, Brookes CL, Robson T, et al. Estrogen receptor and progesterone receptor as predictive biomarkers of response to endocrine therapy: a prospectively powered pathology study in the Tamoxifen and Exemestane Adjuvant Multinational trial. J Clin Oncol 2011;29:1531-8.

5. Hammond ME, Hayes DF, Dowsett M, et al. American Society of Clinical Oncology/College Of American Pathologists guideline recommendations for immunohistochemical testing of estrogen and progesterone receptors in breast cancer. J Clin Oncol 2010;28:2784-95.

6. Allison KH, Hammond MEH, Dowsett M, et al. Estrogen and Progesterone Receptor Testing in Breast Cancer: ASCO/CAP Guideline Update. J Clin Oncol 2020;38:1346-66.

7. Chen T, Zhang N, Moran MS, et al. Borderline ERPositive Primary Breast Cancer Gains No Significant Survival Benefit From Endocrine Therapy: A Systematic Review and Meta-Analysis. Clin Breast Cancer 2018;18:1-8.

8. Bouchard-Fortier A, Provencher L, Blanchette C, et al. Prognostic and predictive value of low estrogen receptor expression in breast cancer. Curr Oncol 2017;24:e106-14.

9. Fujii T, Kogawa T, Dong W, et al. Revisiting the definition of estrogen receptor positivity in HER2-negative primary breast cancer. Ann Oncol 2017;28:2420-8. 
10. Sheffield BS, Kos Z, Asleh-Aburaya K, et al. Molecular subtype profiling of invasive breast cancers weakly positive for estrogen receptor. Breast Cancer Res Treat 2016;155:483-90.

11. Cheang MC, Martin M, Nielsen TO, et al. Defining breast cancer intrinsic subtypes by quantitative receptor expression. Oncologist 2015;20:474-82.

12. Yi M, Huo L, Koenig KB, et al. Which threshold for ER positivity? a retrospective study based on 9639 patients. Ann Oncol 2014;25:1004-11.

13. Prabhu JS, Korlimarla A, Desai K, et al. A Majority of Low (1-10\%) ER Positive Breast Cancers Behave Like Hormone Receptor Negative Tumors. J Cancer 2014;5:156-65.

14. Balduzzi A, Bagnardi V, Rotmensz N, et al. Survival outcomes in breast cancer patients with low estrogen/ progesterone receptor expression. Clin Breast Cancer 2014;14:258-64.

15. Deyarmin B, Kane JL, Valente AL, et al. Effect of ASCO/ CAP guidelines for determining ER status on molecular subtype. Ann Surg Oncol 2013;20:87-93.

16. Iwamoto T, Booser D, Valero V, et al. Estrogen receptor (ER) mRNA and ER-related gene expression in breast cancers that are $1 \%$ to $10 \%$ ER-positive by immunohistochemistry. J Clin Oncol 2012;30:729-34.

17. Raghav KP, Hernandez-Aya LF, Lei X, et al. Impact of low estrogen/progesterone receptor expression on survival outcomes in breast cancers previously classified as triple negative breast cancers. Cancer 2012;118:1498-506.

doi: $10.21037 /$ tbcr-21-12

Cite this article as: Jia Z, Niu S, Wang X, Wu S, Li J, Han M, Liu Y. Development and validation of a nomogram for predicting the status of estrogen receptor-low-positive breast cancer. Transl Breast Cancer Res 2021;2:23.
18. Landmann A, Farrugia DJ, Zhu L, et al. Low Estrogen Receptor (ER)-Positive Breast Cancer and Neoadjuvant Systemic Chemotherapy: Is Response Similar to Typical ER-Positive or ER-Negative Disease? Am J Clin Pathol 2018;150:34-42.

19. Benefield HC, Allott EH, Reeder-Hayes KE, et al. Borderline Estrogen Receptor-Positive Breast Cancers in Black and White Women. J Natl Cancer Inst 2020;112:728-36.

20. Dixon JM, Cameron DA, Arthur LM, et al. Accurate Estrogen Receptor Quantification in Patients with Negative and Low-Positive Estrogen-Receptor-Expressing Breast Tumors: Sub-Analyses of Data from Two Clinical Studies. Adv Ther 2019;36:828-41.

21. Chia SK, Bramwell VH, Tu D, et al. A 50-gene intrinsic subtype classifier for prognosis and prediction of benefit from adjuvant tamoxifen. Clin Cancer Res 2012;18:4465-72.

22. Rawat RR, Ruderman D, Macklin P, et al. Correlating nuclear morphometric patterns with estrogen receptor status in breast cancer pathologic specimens. NPJ Breast Cancer 2018;4:32.

23. Pruneri G, Vingiani A, Denkert C. Tumor infiltrating lymphocytes in early breast cancer. Breast 2018;37:207-14.

24. Hamy AS, Bonsang-Kitzis H, De Croze D, et al. Interaction between Molecular Subtypes and Stromal Immune Infiltration before and after Treatment in Breast Cancer Patients Treated with Neoadjuvant Chemotherapy. Clin Cancer Res 2019;25:6731-41. 